\title{
Tissue Reactivity of the 14F7 Mab Raised against N-Glycolyl GM3 Ganglioside in Tumors of Neuroectodermal, Mesodermal, and Epithelial Origin
}

\author{
Rancés Blanco, ${ }^{1}$ Yisel Quintana, ${ }^{1}$ Damián Blanco, ${ }^{2}$ Mercedes Cedeño, ${ }^{1}$ Charles E. Rengifo, ${ }^{3}$ \\ Milagros Frómeta, ${ }^{1}$ Martha Ríos, ${ }^{4}$ Enrique Rengifo, ${ }^{1}$ and Adriana Carr ${ }^{5}$ \\ ${ }^{1}$ Department of Quality Control, Center of Molecular Immunology, 216 Street and 15 Avenue Atabey, Playa, P.O. Box 16040, \\ 11600 Havana, Cuba \\ ${ }^{2}$ Department of Cell Biology and Tissues Banking, National Institute of Oncology and Radiobiology, 29 and F Street Vedado, \\ Plaza de la Revolución, 10400 Havana, Cuba \\ ${ }^{3}$ Department of Pathology, Manuel Fajardo General Hospital, Zapata and D Street Vedado, Plaza de la Revolución, \\ 10400 Havana, Cuba \\ ${ }^{4}$ Department of Neurosurgery, Juan Manuel Marquez Pediatric Hospital, Marianao, 11400 Havana, Cuba \\ ${ }^{5}$ Research and Development Direction, Center of Molecular Immunology, 216 Street and 15 Avenue Atabey, Playa, \\ P.O. Box 16040, 11600 Havana, Cuba
}

Correspondence should be addressed to Rancés Blanco; rances@cim.sld.cu

Received 27 August 2012; Accepted 20 December 2012

Academic Editor: George T. Tsangaris

Copyright (C) 2013 Rancés Blanco et al. This is an open access article distributed under the Creative Commons Attribution License, which permits unrestricted use, distribution, and reproduction in any medium, provided the original work is properly cited.

The expression of N-glycolylneuraminic acid forming the structure of gangliosides and/or other glycoconjugates (HanganutziuDeicher antigen) in human has been considered as a tumor-associated antigen. Specifically, some reports of 14F7 Mab (a highly specific Mab raised against N-glycolyl GM3 ganglioside) reactivity in human tumors have been recently published. Nevertheless, tumors of epithelial origin have been mostly evaluated. The goal of the present paper was to evaluate the immunohistochemical recognition of 14F7 Mab in different human tumors of neuroectodermal, mesodermal, and epithelial origins using an immunoperoxidase staining method. Samples of fetal, normal, and reactive astrocytosis of the brain were also included in the study. In general, nontumoral tissues, as well as, low-grade brain tumors showed no or a limited immunoreaction with 14F7 Mab. Nevertheless, high-grade astrocytomas (III-IV) and neuroblastomas, as well as, sarcomas and thyroid carcinomas were mostly reactive with 14F7. No reaction was evidenced in medulloblastomas and ependymoblastomas. Our data suggest that the expression of N-glycolyl GM3 ganglioside could be related to the aggressive behavior of malignant cells, without depending on the tumor origin. Our data could also support the possible use of N-glycolyl GM3 as a target for both active and passive immunotherapies of malignancies expressing this molecule.

\section{Introduction}

Changes in the composition of cell surface glycolipids that take place during malignant transformation have been extensively described [1]. Particularly, numerous studies on glycolipids have been focused on gangliosides [2]. Gangliosides are glycosphingolipids containing sialic acid engaged in a wide variety of biological events that occur at vertebrate's cell membrane. They are widely distributed in both normal and tumoral human tissues of neuroectodermal origin $[3,4]$.

The most abundant sialic acid variants in mammals are $\mathrm{N}$-acetylneuraminic acid (NeuAc) and N-glycolylneuraminic acid (NeuGc). NeuAc acid is the predominant sialic acid species expressed in mammalian brain gangliosides. Whereas, NeuGc is a predominant sialic acid species expressed in gangliosides from nonneural tissues of most nonhuman 
species $[5,6]$. In contrast to NeuAc, the expression of the $\mathrm{NeuGc}$ forming the structure of gangliosides and/or other glycoconjugates (Hanganutziu-Deicher antigen) in human has been considered as a tumor-associated antigen [7]. The only structural difference between NeuAc and NeuGc is a single oxygen atom at the C-5 position of NeuGc catalyzed by the cytidine monophospho- $\mathrm{N}$-acetylneuraminic acid hydroxylase (CMP-NeuAc hydroxylase) [8]. This minor difference is able to induce an immune response [9] as well as to develop specific antibodies raised against $\mathrm{N}$-glycolylated gangliosides [10, 11].

The aberrant expression of the NeuGc residues in humans has been considered to be associated with the altered metabolism of malignant cells [9, 12, 13]. Normal human cells are incapable of synthesizing NeuGc due to a specific inactivating mutation in the CMP-NeuAc hydroxylase gene [14]. However, some authors have suggested an alternative pathway to the NeuGc synthesis from other intermediates of cellular metabolism in some human tumors [9].

Recently, some reports of 14F7 Mab (a highly specific Mab raised against $\mathrm{N}$-glycolyl GM3 ganglioside) reactivity in formalin-fixed and paraffin-embedded tissues have been published. Nevertheless, epithelial-derived tumors have been mostly evaluated [15-19]. In this way, the analysis of NeuGcGM3 expression in different human neoplasms could be useful as a better basis for understanding the molecular pathogenesis of malignancies as well as to extend the assessment of this molecule as target for cancer immunotherapy. For these reasons, in this work was evaluated the recognition of 14F7 Mab in a serie of neuroectodermal, mesodermal, and epithelial derived tumors. Samples of fetal, normal, and reactive astrocytosis were also included in the study.

\section{Materials and Methods}

2.1. Monoclonal Antibody. We used the 14F7 Mab (IgG1) a highly specific anti-NeuGcGM3 ganglioside antibody. This Mab was generated by immunization of Balb/c mice with NeuGcGM3 hydrophobically conjugated with human very low-density lipoproteins (VLDLs) adjuvated with Complete Freud adjuvant (CFA). Afterward, 14F7 Mab was obtained by the hybridoma resulting in the fusion of spleen cells with mouse myeloma cell line P3X63Ag653 as described in [10].

2.2. Tissue Specimens. Routinely processed, formalin-fixed, and paraffin-embedded archival samples with diagnosis of fetal tissues (3), normal adult tissues (10), reactive astrocytosis of the brain (3), pediatric brain tumors (35), sarcomas (30), and thyroid carcinomas (25) as well as frozen adult tissues (84 normal and 11 tumoral) were received from the pathology departments of Ramón González Coro Gyneco-Obstetric Hospital, Juan Manuel Márquez Pediatric Hospital, the legal-medicine department at "Amalia Simoni" Provincial Hospital of Camagüey, the National Institute of Neurology and Neuropathology, and the National Institute of Oncology and Radiobiology. Fetal tissues were obtained from 19-week-old fetus aborted by Rivanol, and normal tissues were removed at autopsy of healthy persons having suffered clinical death or by conventional intraoperatory biopsy. All samples were used after obtaining an approved consent by the institutional ethical committees.

2.3. Previous Processing. For fresh samples, tissues were frozen in liquid nitrogen and stored at $-70^{\circ} \mathrm{C}$ until sectioning. Then, five micrometres sections were obtained in a cryostat and slides were stored at $-20^{\circ} \mathrm{C}$ until they were used. These sections were fixed in $4 \%$ paraformaldehyde during 20 minutes at room temperature. All samples were washed in tap water and rehydrated in distilled water for 10 minutes and TBS for 5 minutes. Slides were incubated with biotinblocking system (X0560, Dako, Denmark A/S), according to manufacture instructions. Afterward, tissue samples were washed with TBS during $10 \mathrm{~min}$. For formalin-fixed and paraffin-embedded tissues, five micrometer serial sections from each block were obtained, and the slides were processed as it was previously described [16].

2.4. Immunohistochemical Staining and Evaluation. The method previously described in [16] was used. Briefly, the samples were incubated with 14F7 Mab followed by a peroxidase avidin-biotin system. Negative controls were performed substituting primary antibody for washing buffer (TBS). Colonic adenocarcinoma [16] and a breast infiltrating carcinoma [10] were taken as positive control for both paraffin-embedded and frozen tissues, respectively. Enzymatic activity was visualized with a DAB (K3465, Dako, Denmark A/S) solution, and slides were counterstained with Mayer's Hematoxylin (S2020, Dako, Denmark A/S). Staining of both cell membrane and cytoplasm was considered as positive for 14F7 Mab. A semiquantitative scoring system was used to define levels of reactivity. According to the staining pattern, the tumor sample was assigned to 1 of 4 scores: 0 , no staining; 1, weak staining; 2, moderate staining; and 3, strong staining of malignant cells. All microscopic analyses were performed by two different observers.

\section{Results}

3.1. A Limited Reaction of $14 F 7$ Mab Was Detected in Nontumoral Tissues. No reaction was observed with the 14F7 Mab in fetal tissues, except for a weak-to-moderate reactivity in less than $25 \%$ of brain neurons in $1 / 3$ cases (Table 1). No staining was evidenced neither in formalinfixed and paraffin-embedded (0/10) nor frozen (1/84) normal tissues, except for a weak reaction of mucous cells from small intestine (1/3) (Table 2). Similar results were obtained from reactive astrocytosis of the brain $(0 / 3)$.

\subsection{F7 Mab Staining Was Mainly Found in Frozen Tumors} with Astrocytic Differentiation. An intense homogeneous and finely granular pattern of recognition was detected in $2 / 2$ anaplastic astrocytomas, 1/2 oligoastrocytic tumors, while the glioblastoma (0/1) showed no reaction with 14F7 Mab. The reaction was mainly located on the plasmatic membrane and in more than $50 \%$ of malignant astrocytes. No staining 
TABLE 1: Immunorecognition of 14F7 Mab in formalin-fixed and paraffin-embedded nontumoral tissues.

\begin{tabular}{lccc}
\hline Samples & \multicolumn{3}{c}{ Score } \\
\hline $\begin{array}{l}\text { Central nervous system } \\
\quad \text { Fetal }\end{array}$ & 0 & 1 & Total (\%) \\
$\quad$ Neurons & 2 & 1 & $1 / 3(33.33)$ \\
$\quad$ Glias & 3 & 0 & $0 / 3$ \\
$\quad$ Normal & & & \\
$\quad$ Brain & $3^{*}$ & 0 & $0 / 3$ \\
$\quad$ Cerebellum & 4 & 0 & $0 / 4$ \\
$\quad$ Reactive astrocytosis & 3 & 0 & $0 / 3$ \\
Normal thyroid & 3 & 0 & $0 / 3$ \\
\hline
\end{tabular}

0: no staining; 1 : weak staining; ${ }^{*}$ samples from the normal tissue located in peritumoral area.

was observed in oligodendrogliomas (0/3) and meningiomas $(0 / 3)$ (Table 3).

3.3. The Reaction with 14F7 Mab Was Also Evidenced in Formalin-Fixed and Paraffin-Embedded Tumors. Pilocytic astrocytomas (0/4) were not recognized by 14F7 Mab. In contrast, $3 / 5(60.00 \%), 5 / 8(62.50 \%)$ of diffuse and anaplastic astrocytomas; and $2 / 2$ of glioblastomas were moderateto-intense reactive with $14 \mathrm{~F} 7 \mathrm{Mab}$ in more than $50 \%$ of malignant cells (Table 4, Figure 1). One case of anaplastic astrocytoma and the oligodendroglioma showed a weakto-moderate and finely granular reactivity mainly located in plasmatic membrane and also in the cytoplasm of less than $25 \%$ of malignant cells. No immunorecognition was observed in ependymomas, while 14F7 Mab was moderateto-intense reactive at less than $25 \%$ of malignant cells in $1 / 5$ anaplastic ependymomas. In the other hand, neuroblastomas $(2 / 3)$ and ganglioneuroblastomas (2/2) exhibited a moderateto-intense reaction in both the plasmatic membrane and the cytoplasm of more than $25 \%$ of malignant cells. Concerning to medulloblastomas (0/2) and ependymoblastomas (0/2) no immunostaining was evidenced.

3.4. Sarcomas Were Also Recognized by $14 F 7$ Mab. The 14F7 Mab reactivity was detected in $25 / 30(83.3 \%)$ of all studied soft tissue and bone sarcomas depending without the histopathological subtype. Considering the different histological subtypes of sarcomas, $8 / 8$ muscular sarcomas, $3 / 3$ vascular sarcomas, $2 / 2$ peripheral nerve sheath tumours, $7 / 10$ other fusocellular sarcomas, $2 / 3$ liposarcomas, and $3 / 3$ osteosarcomas were recognized by 14F7 Mab. No immunoreaction was evidenced in the synovial sarcoma (Table 5).

In general, the staining with $14 \mathrm{~F} 7$ was observed as a finely granular reaction mainly located in the cell membrane but also in the cytoplasm of more than $50 \%$ of malignant cells (Figure 2). Almost all sarcomas were moderate-to-intense reactive with $14 \mathrm{~F} 7$ although a weak intensity of staining was observed in a low-grade leiomyosarcoma.

3.5. Thyroid Carcinoma. A moderate-to-intense reactivity in 23/25 (92.0\%) of thyroid carcinoma was detected (Table 6).
TABLE 2: Reactivity of 14F7 Mab in frozen normal tissues.

\begin{tabular}{|c|c|c|}
\hline \multirow{2}{*}{ Samples } & \multicolumn{2}{|c|}{ 14F7 Mab reactivity } \\
\hline & No. cases/total & Intensity of reaction \\
\hline Skin & $0 / 3$ & - \\
\hline \multicolumn{3}{|l|}{ Gastrointestinal tract } \\
\hline Esophagus & $0 / 3$ & - \\
\hline Estomach & $0 / 3$ & - \\
\hline Small intestine & $1 / 3$ & +++ \\
\hline Large intestine & $0 / 3$ & - \\
\hline Liver & $0 / 3$ & - \\
\hline Pancreas & $0 / 3$ & - \\
\hline \multicolumn{3}{|l|}{ Respiratory tract } \\
\hline Lung & $0 / 4$ & - \\
\hline \multicolumn{3}{|l|}{ Urogenital tract } \\
\hline Testis & $0 / 4$ & - \\
\hline Prostate & $0 / 3$ & - \\
\hline Kidney & $0 / 4$ & - \\
\hline Urinary bladder & $0 / 3$ & - \\
\hline Ureter & $0 / 3$ & - \\
\hline \multicolumn{3}{|l|}{ Nervous system } \\
\hline Brain & $0 / 4$ & - \\
\hline Cerebellum & $0 / 4$ & - \\
\hline Brain stem & $0 / 3$ & - \\
\hline Spinal cord & $0 / 1$ & - \\
\hline Peripheral nervous & $0 / 1$ & - \\
\hline \multicolumn{3}{|l|}{ Immune system } \\
\hline Thymus & $0 / 1$ & - \\
\hline Tonsils & $0 / 1$ & - \\
\hline Lymph node & $0 / 2$ & - \\
\hline Spleen & $0 / 3$ & - \\
\hline \multicolumn{3}{|l|}{ Cardiovascular system } \\
\hline Heart & $0 / 3$ & - \\
\hline Artery & $0 / 4$ & - \\
\hline Vein & $0 / 4$ & - \\
\hline \multicolumn{3}{|l|}{ Muscle } \\
\hline Estriated & $0 / 4$ & - \\
\hline Smooth & $0 / 4$ & - \\
\hline \multicolumn{3}{|l|}{ Endocrine system } \\
\hline Pituitary & $0 / 2$ & - \\
\hline Thyroid & $0 / 1$ & - \\
\hline
\end{tabular}

$(-)$ : negative; (+++): strong staining of cells.

The reaction with 14F7 showed a finely granular pattern localized in both the plasmatic membrane and the cytoplasm of neoplastic cells (Figure 3).

\section{Discussion}

Unusual glycosylated or sialylated gangliosides have been identified with monoclonal antibodies generated against tumor-associated antigens, and they were considered as targets for use in passive and active immunotherapy of some malignant neoplasms $[10,11]$. Between them, the expression 


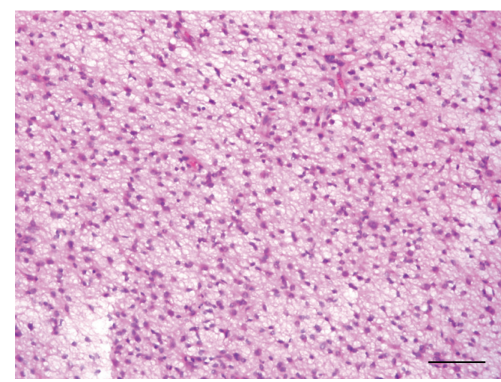

(a)

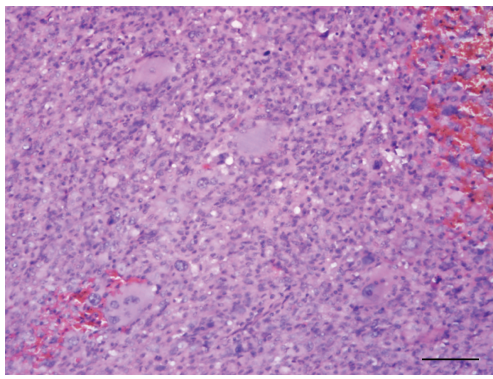

(c)

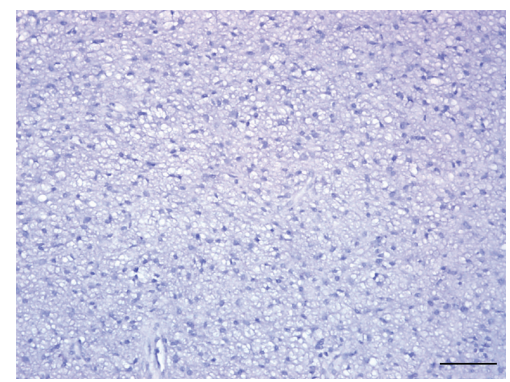

(b)

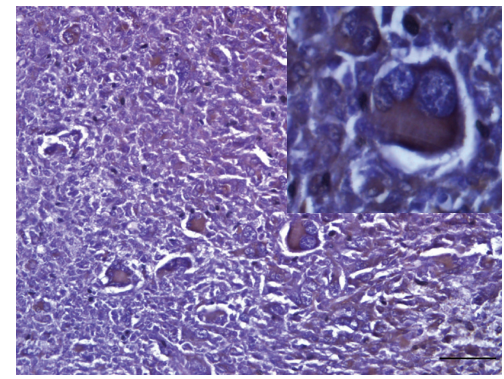

(d)

Figure 1: Hematoxylin and eosin staining of pilocytic astrocytoma (a) and glioblastoma (c). See no reaction of 14F7 Mab in pilocytic astrocytoma (b) while an intense reactivity with 14F7 Mab was observed in glioblastoma cells (d). The staining was located on both cell membrane and cytoplasm of malignant cells (inset on the upper-right corner, 400x magnification). Black bar $=100 \mu \mathrm{m}$.

TABLE 3: Immunostaining of 14F7 Mab in frozen tumors from central nervous system.

\begin{tabular}{lllc}
\hline Histopathological type $^{*}$ & 0 & 3 & Total (\%) \\
\hline Anaplastic astrocytoma & 0 & 2 & $2 / 2(100)$ \\
Glioblastoma & 1 & 0 & $0 / 1$ \\
Oligodendroglioma & 3 & 0 & $0 / 3$ \\
Oligoastrocytoma & 1 & 1 & $1 / 2(50)$ \\
Meningioma & 3 & 0 & $0 / 3$ \\
\hline
\end{tabular}

${ }^{*}$ WHO grade classification. 0: no reaction; 3: strong staining of malignant cells.

of a nonhuman sialic acid ( $\mathrm{N}$-glycolylneuraminic) forming the structure of gangliosides and/or other glycoconjugates has been considered one of the most important antigens $[1,20]$.

The structural difference between $\mathrm{N}$-acetylneuraminic (normal constituent of human tissues) and $\mathrm{N}$-glycolylneuraminic (tumor-associated antigen) is crucial in many aspects of the cellular behavior $[12,21]$ and has permitted the development of specific antibodies raised against the Hanganutziu-Deicher (HD) antigen or N-glycolylated gangliosides as well as their immunohistochemical evaluation using both frozen and formalin-fixed and paraffin-embedded tissues $[9,22]$. The antigenic determinant of HD antigen is $\mathrm{N}$-glycolylneuraminic acid. Therefore, HD is classified as a heterophile antigen and chemically defined as a glycolipid and/or glycoprotein (glycoconjugates) which contains NeuGc. This antigen has been reported to be almost absent in normal human tissues but can be expressed on a variety of human malignant cells [23].

In our study, both frozen and formalin-fixed and paraffinembedded nontumoral human tissues were not reactive with 14F7 Mab (IgG1 highly specific against N-glycolyl GM3 ganglioside), except for a weak-to-moderate staining of some neurons in fetal tissues. Additionally, we observed an intense staining of 14F7 in mucous cells from small intestine. In previous studies, normal eukaryotic cells were able to take in a portion of ingested NeuGc and process it for their own glycoconjugates $[13,20]$. In line with this, small levels of expression of NeuGc have been found in some normal human tissues (e.g., epithelial cells and their secretions) [12]. The limited reactivity of 14F7 Mab in nontumoral tissues confirmed that $14 \mathrm{~F} 7 \mathrm{Mab}$ is able to distinguish between the $\mathrm{N}$-glycolyl and the $\mathrm{N}$-acetyl functions of the GM3 ganglioside [10]. Furthermore, the limited recognition of 14F7 Mab in other normal tissues has been also reported by our group [1619].

In contrast, our group reported the expression of NeuGcGM3 in breast tumors using both thin layer chromatography (TLC) immunostaining and fast atom bombardment mass spectroscopy (FAB/MS) analysis [24]. In addition, we published the immunohistochemical recognition of the 14F7 Mab in breast infiltrating ductal carcinoma and melanoma by immunohistochemistry using frozen tissues fixed in $4 \%$ paraformaldehyde. This finding suggested that the structure recognized in breast tumors could be the oligosaccharide core of NeuGcGM3 present in glycoconjugates [10]. The in vivo tissular expression of NeuGcGM3 was also confirmed by the radioimmunoscintigrafic technique using 
TABLE 4: Recognition of 14F7 Mab in primary nervous system tumors of pediatrics patients.

\begin{tabular}{|c|c|c|c|c|c|}
\hline \multirow{2}{*}{ Histopathological type ${ }^{*}$} & \multicolumn{5}{|c|}{ Score } \\
\hline & 0 & 1 & 2 & 3 & Total (\%) \\
\hline Pilocytic astrocytoma & 4 & 0 & 0 & 0 & $0 / 4$ \\
\hline Diffuse astrocytoma & 2 & 0 & 1 & 2 & $3 / 5(60.00)$ \\
\hline Anaplastic astrocytoma & 3 & 1 & 1 & 3 & $5 / 8(62.50)$ \\
\hline Glioblastoma & 0 & 0 & 0 & 2 & $2 / 2$ \\
\hline Oligodendroglioma & 0 & 1 & 0 & 0 & $1 / 1$ \\
\hline Ependymoma & 1 & 0 & 0 & 0 & $0 / 1$ \\
\hline Anaplastic ependymoma & 4 & 0 & 1 & 0 & $1 / 5(20.00)$ \\
\hline Medulloblastoma & 2 & 0 & 0 & 0 & $0 / 2$ \\
\hline Neuroblastoma & 1 & 0 & 0 & 2 & $2 / 3(66.66)$ \\
\hline Ganglioneuroblastoma & 0 & 0 & 2 & 0 & $2 / 2$ \\
\hline Ependymoblastoma & 2 & 0 & 0 & 0 & $0 / 2$ \\
\hline
\end{tabular}

${ }^{*}$ WHO grade classification. 0: no staining; 1: weak staining; 2: moderate staining; 3: strong staining of malignant cells.

TABLE 5: Immunoreactivity of 14F7 Mab in sarcomas.

\begin{tabular}{|c|c|c|c|c|c|}
\hline \multirow{2}{*}{ Histopathological type } & \multicolumn{5}{|c|}{ Score } \\
\hline & 0 & 1 & 2 & 3 & No. cases \\
\hline \multicolumn{6}{|l|}{ Soft tissue sarcoma } \\
\hline Leiomyosarcoma & 0 & 1 & 2 & 4 & $7 / 7$ \\
\hline Rhabdomyosarcoma & 0 & 0 & 0 & 1 & $1 / 1$ \\
\hline Endoteliosarcoma & 0 & 0 & 0 & 1 & $1 / 1$ \\
\hline Hemangiopericytoma & 0 & 0 & 0 & 2 & $2 / 2$ \\
\hline Neurofibrosarcoma & 0 & 0 & 0 & 1 & $1 / 1$ \\
\hline Malignant schwannoma & 0 & 0 & 0 & 1 & $1 / 1$ \\
\hline Fibrosarcoma & 2 & 0 & 0 & 1 & $1 / 3$ \\
\hline Malignant fibrous histiocytoma & 1 & 0 & 0 & 3 & $3 / 4$ \\
\hline NOS (not otherwise specified) & 0 & 0 & 0 & 3 & $3 / 3$ \\
\hline Liposarcoma & 1 & 0 & 0 & 2 & $2 / 3$ \\
\hline Synovial sarcoma & 1 & 0 & 0 & 0 & $0 / 1$ \\
\hline \multicolumn{6}{|l|}{ Nonsoft tissue sarcoma } \\
\hline Osteosarcoma & 0 & 0 & 0 & 3 & $3 / 3$ \\
\hline Total & & & & & $25 / 30(83.3 \%)$ \\
\hline
\end{tabular}

NOS: not otherwise specified. 0: no staining; 1: weak staining; 2: moderate staining; 3: strong staining of malignant cells.

14F7 Mab labelled with 99mTc [25]. Afterward, we reported the immunohistochemical reactivity of $14 \mathrm{~F} 7$ in a variety of formalin-fixed and paraffin-embedded tumor tissues, despite the extraction of glycolipids during the routine histological procedures [16-19].

Recently, Scursoni et al. reported the recognition of 14F7 Mab in pediatric neuroblastoma using formalin-fixed and paraffin-embedded tissues and suggested that the expression of NeuGcGM3 ganglioside is preserved in the more aggressive tumors. Moreover, a clinical trial with Racotumomab (anti-idiotypic vaccine) in pediatric neuroectodermal tumors has been suggested in [26]. In this study, we describe the reactivity of the $14 \mathrm{~F} 7$ in tumors of the central nervous system using both frozen tissues after $4 \%$ paraformaldehyde fixation and formalin-fixed and paraffin-embedded tissues. Malignancies with astrocytic differentiation and among them: diffuse and anaplastic astrocytomas, glioblastomas, an neuroblastomas were mainly recognized by $14 \mathrm{~F} 7 \mathrm{Mab}$. Our data seems to be also in agreement with the preferential reactivity of 14F7 Mab in more aggressive types of human astrocytoma. In line with this, the progression of malignant brain tumors has been associated with altered gangliosides composition and distribution [2].

On the other hand, a preliminary study about the reactivity of 14F7 in Ewing sarcoma has been previously described suggesting the potential use of NeuGcGM3 for cancer immunotherapy [26]. Here, we obtained the reactivity of 14F7 Mab in almost all soft and nonsoft tissues sarcomas without taking into account the histopathological classification. At present, a lot of studies are focused to better understand the molecular pathogenesis of sarcomas as well as the identification of reliable molecular markers and possible therapeutic targets. Some of these studies have been focused in sialic acid content [27]. Authors have reported 


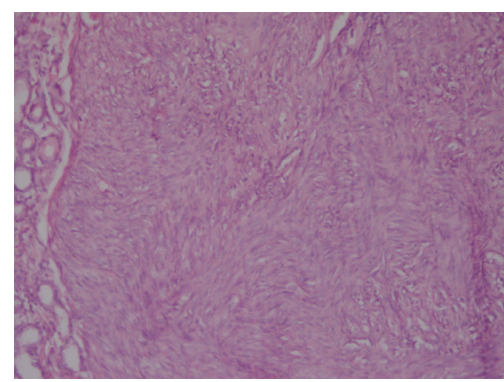

(a)

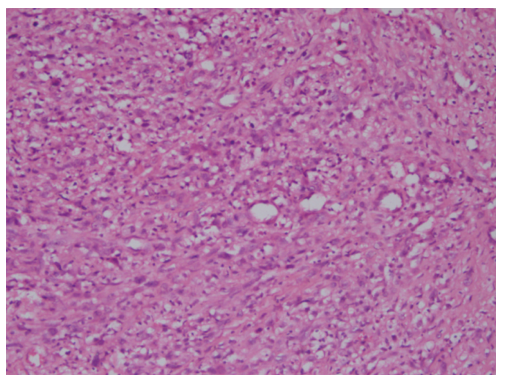

(c)

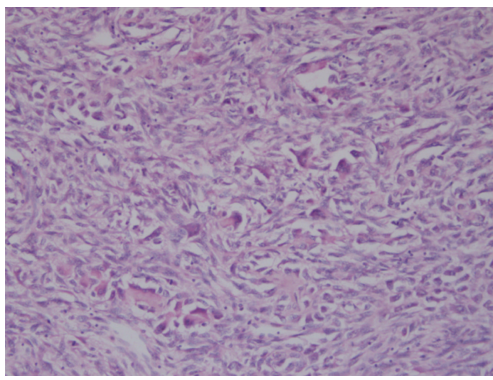

(e)

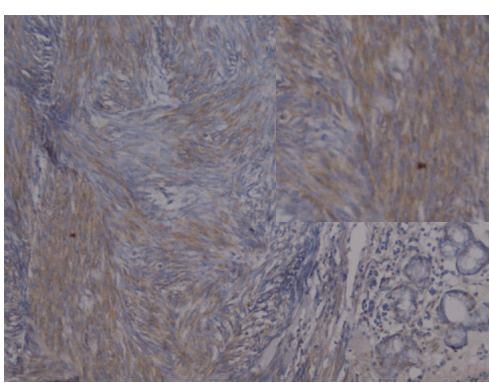

(b)

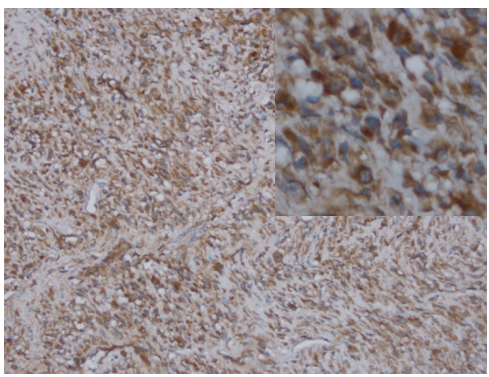

(d)

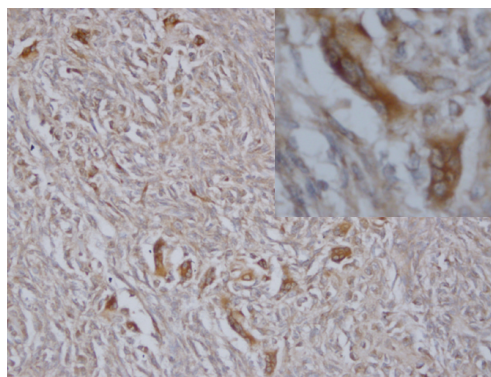

(f)

FIGURE 2: Hematoxylin and eosin staining of low-grade leiomyosarcoma of the stomach (a), malignant fibrous histiocytoma (c), and osteogenic sarcoma of the breast (e). Moderate reaction of 14F7 Mab in malignant smooth muscle cells. See no recognition of 14F7 in normal glands of the stomach (b). An intense immunostaining with 14F7 Mab located on both cell membrane and cytoplasm was detected in malignant fibrous histiocytoma (d) and osteoid cells (f) (inset on the upper-right corner, 400x magnification). Black bar $=100 \mu \mathrm{m}$.

TABLE 6: Staining of 14F7 Mab in thyroid carcinomas.

\begin{tabular}{llccc}
\hline Histopathological type & & Score & 3 & No. cases (\%) \\
\hline Thyroid carcinoma & 0 & 2 & & 11 \\
$\quad$ Papillary & 1 & 0 & 9 & $11 / 12$ \\
$\quad$ Follicular & 1 & 1 & 1 & $2 / 2$ \\
$\quad$ Medullary & 0 & 1 & 21 & $23 / 25(92.0 \%)$ \\
\hline Total & 2 & 2 & & $2 / 11$ \\
\hline
\end{tabular}

${ }^{*}$ WHO grade classification. 0: no reaction; 2: moderate staining; and 3: strong staining of malignant cells.

increased amount of serum total sialic acid as well as the detection of $\mathrm{N}$-glycolylneuraminic acid antibody in patient bearing sarcomas [28]. Interestingly, our group has evidence about the occurrence of higher levels of antibodies raised against NeuGcGM3 in patients bearing sarcomas (Carr A, unpublished data).
Neoplastic transformation of the thyroid gland has been reported to be accompanied by changes in cellular sialylation. A limited or absent expression of sialic acid in the surface of follicular cells in normal thyroid glands, adenomas, and goiters has been demonstrated. In contrast, a weak-to-intense positivity for sialic acid was found in thyroid carcinomas [29]. 


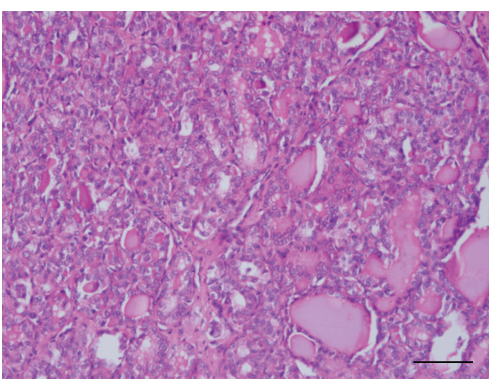

(a)

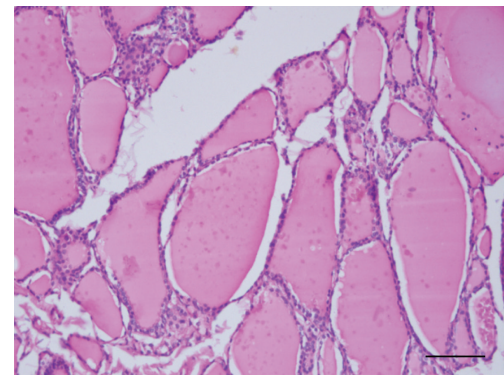

(c)

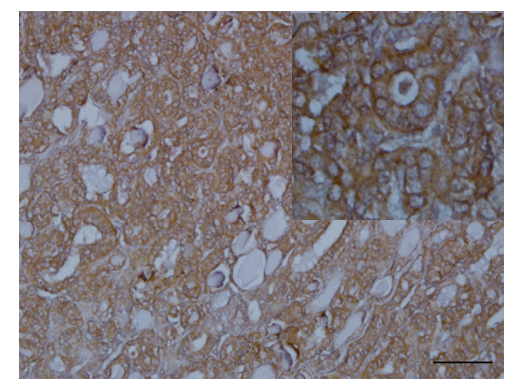

(b)

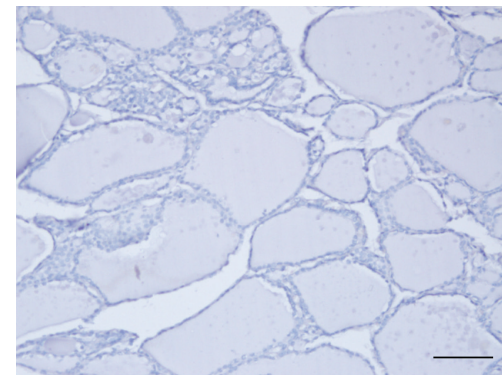

(d)

FIGURE 3: Hematoxylin and eosin staining of thyroid papillary carcinoma (a) and nontumoral adjacent area (c). Strong and finely granular reactivity of $14 \mathrm{~F} 7 \mathrm{Mab}(\mathrm{b})$. The reaction was mainly located on the plasmatic membrane of malignant cells (inset on the upper-right corner, 400x magnification). Note no reaction of $14 \mathrm{~F} 7$ in nontumoral thyroid tissue (d). Black bar $=100 \mu \mathrm{m}$.

In this study, we reported the immunohistochemical recognition of 14F7 Mab in the majority of thyroid carcinomas but not in their normal counterpart. In this way, our data permit to consider the potential use of NeuGcGM3 as recognized by $14 \mathrm{~F} 7$ in both the distinction of malignant from benign thyroid lesions and in being a potential target for active and passive immunotherapy in persistent and recurrent thyroid carcinomas.

Finally, intratumoral hypoxia (low oxygen tension) has been associated with aggressive disease, poor prognosis, and resistance to conventional therapies of malignant brain tumors, sarcomas, and thyroid carcinomas [30-32]. Tumor hypoxia has been considered responsible of NeuGcGM2 ganglioside expression in human cancer cells through the incorporation of NeuGc. The effect of hypoxia could be to expedite sialic acid transport from the external medium, in relation to the increment of sialin expression (a sialic acid molecule transporter) [33]. The role of NeuGcGM3 in tumoral progression as well as its suppressor properties has been previously reported $[34,35]$. In this way, studies focused on the evaluation of intratumoral hypoxia and NeuGcGM3 relations are being planned in our lab.

\section{Conclusions}

The expression of NeuGcGM3 in neuroectodermal, mesodermal, and epithelial derived tumors but not in normal sections suggests that the expression of this ganglioside could be related to the aggressive behavior of malignant cells, without depending on the tumor origin. Our data could support the possible use of NeuGcGM3 as a target for both active and passive immunotherapies of malignancies expressing this molecule.

\section{Conflict of Interests}

The authors report no conflict of interests.

\section{Acknowledgments}

The authors want to thank the Legal-Medicine Department at "Amalia Simoni" Provincial Hospital of Camagüey as well as the Pathology Departments of Juan Manuel Márquez Pediatric Hospital, National Institute of Neurology and Neuropathology, Ramón González Coro Gyneco-Obstetric Hospital, and National Institute of Oncology and Radiobiology for providing them with the samples of fetal, normal; and pathological tissues. Financial support was provided by the Center of Molecular Immunology.

\section{References}

[1] S. I. Hakomori, "Aberrant glycosylation in cancer cell membranes as focused on glycolipids: overview and perspectives," Cancer Research, vol. 45, no. 6, pp. 2405-2414, 1985.

[2] N. Shinoura, T. Dohi, T. Kondo, M. Yoshioka, K. Takakura, and M. Oshima, "Ganglioside composition and its relation to clinical data in brain tumors," Neurosurgery, vol. 31, no. 3, pp. 541-549, 1992.

[3] T. Yamashita, R. Wada, T. Sasaki et al., "A vital role for glycosphingolipid synthesis during development and differentiation," 
Proceedings of the National Academy of Sciences of the United States of America, vol. 96, no. 16, pp. 9142-9147, 1999.

[4] G. Kohla, E. Stockfleth, and R. Schauer, "Gangliosides with Oacetylated sialic acids in tumors of neuroectodermal origin," Neurochemical Research, vol. 27, no. 7-8, pp. 583-592, 2002.

[5] T. N. Seyfried, R. K. Yu, M. Saito, and M. Albert, "Ganglioside composition of an experimental mouse brain tumor," Cancer Research, vol. 47, no. 13, pp. 3538-3542, 1987.

[6] J. A. Ecsedy, K. A. Holthaus, H. C. Yohe, and T. N. Seyfried, "Expression of mouse sialic acid on gangliosides of a human glioma grown as a xenograft in SCID mice," Journal of Neurochemistry, vol. 73, no. 1, pp. 254-259, 1999.

[7] H. Higashi, Y. Nishi, and Y. Fukuin, "Tumor-associated expression of glycosphingolipid Hanganutziu-Deicher antigen in human cancers," Gann, The Japanese Journal of Cancer Research, vol. 75, no. 11, pp. 1025-1029, 1984.

[8] W. Schlenzka, L. Shaw, S. Kelm et al., "CMP-Nacetylneuraminic acid hydroxylase: the first cytosolic Rieske iron-sulphur protein to be described in Eukarya," FEBS Letters, vol. 385, no. 3, pp. 197-200, 1996.

[9] Y. N. Malykh, R. Schauer, and L. Shaw, "N-Glycolylneuraminic acid in human tumours," Biochimie, vol. 83, no. 7, pp. 623-634, 2001.

[10] A. Carr, A. Mullet, Z. Mazorra et al., "A mouse IgG1 monoclonal antibody specific for N-glycolyl GM3 ganglioside recognized breast and melanoma tumors," Hybridoma, vol. 19, no. 3 , pp. 241-247, 2000.

[11] A. M. Vazquez, M. Alfonso, B. Lanne et al., "Generation of a murine monoclonal antibody specific for $\mathrm{N}$-glycolylneuraminic acid-containing gangliosides that also recognizes sulfated glycolipids," Hybridoma, vol. 14, no. 6, pp. 551-556, 1995.

[12] P. Tangvoranuntakul, P. Gagneux, S. Diaz et al., "Human uptake and incorporation of an immunogenic nonhuman dietary sialic acid," Proceedings of the National Academy of Sciences of the United States of America, vol. 100, no. 21, pp. 12045-12050, 2003.

[13] M. Bardor, D. H. Nguyen, S. Diaz, and A. Varki, "Mechanism of uptake and incorporation of the non-human sialic acid Nglycolylneuraminic acid into human cells," Journal of Biological Chemistry, vol. 280, no. 6, pp. 4228-4237, 2005.

[14] H. H. Chou, H. Takematsu, S. Diaz et al., "A mutation in human CMP-sialic acid hydroxylase occurred after the HomoPan divergence," Proceedings of the National Academy of Sciences of the United States of America, vol. 95, no. 20, pp. 11751-11756, 1998.

[15] H. van Cruijsen, M. Ruiz, P. van der Valk, T. D. de Gruijl, and G. Giaccone, "Tissue micro array analysis of ganglioside $\mathrm{N}$-glycolyl GM3 expression and signal transducer and activator of transcription (STAT)-3 activation in relation to dendritic cell infiltration and microvessel density in non-small cell lung cancer," BMC Cancer, vol. 9, article 180, 2009.

[16] R. Blanco, E. Rengifo, M. Cedeño, C. E. Rengifo, D. F. Alonso, and A. Carr, "Immunoreactivity of the 14F7 Mab raised against $\mathrm{N}$-Glycolyl GM3 ganglioside in epithelial malignant tumors from digestive system," ISRN Gastroenterology, vol. 2011, Article ID 645641, 8 pages, 2011.

[17] R. Blanco, E. Rengifo, Ch. E. Rengifo, M. Cedeño, M. Frómeta, and A. Carr, "Immunohistochemical reactivity of the 14F7 monoclonal antibody raised against N-glycolyl GM3 ganglioside in some benign and malignant skin neoplasms," ISRN Dermatology, vol. 2011, Article ID 848909, 8 pages, 2011.
[18] R. Blanco, M. Cedeño, X. Escobar et al., "Immunorecognition of the 14F7 Mab raised against N-Glycolyl GM3 ganglioside in some normal and malignant tissues from genitourinary system," ISRN Pathology, vol. 2011, Article ID 953803, 10 pages, 2011.

[19] R. Blanco, Ch. E. Rengifo, M. Cedeño, M. Frómeta, E. Rengifo, and A. Carr, "Immunoreactivity of the 14F7 Mab (raised against $\mathrm{N}$-glycolyl GM3 ganglioside) as a positive prognostic factor in non-small-cell lung cancer," Pathology Research International, vol. 2012, Article ID 235418, 12 pages, 2012.

[20] C. Oetke, S. Hinderlich, R. Brossmer, W. Reutter, M. Pawlita, and O. T. Keppler, "Evidence for efficient uptake and incorporation of sialic acid by eukaryotic cells," European Journal of Biochemistry, vol. 268, no. 16, pp. 4553-4561, 2001.

[21] T. Kawai, A. Kato, H. Higashi, S. Kato, and M. Naiki, "Quantitative determination of $\mathrm{N}$-glycolylneuraminic acid expression in human cancerous tissues and avian lymphoma cell lines as a tumor-associated sialic acid by gas chromatography-mass spectrometry," Cancer Research, vol. 51, no. 4, pp. 1242-1246, 1991.

[22] H. Higashi, M. Naiki, S. Matuo, and K. Okouchi, "Antigen of "serum sickness" type of heterophile antibodies in human sera: identification as gangliosides with N-glycolylneuraminic acid," Biochemical and Biophysical Research Communications, vol. 79, no. 2, pp. 388-395, 1977.

[23] T. Nishimaki, K. Kano, and F. Milgrom, "Hanganutziu-Deicher antigen and antibody in pathologic sera and tissues," Journal of Immunology, vol. 122, no. 6, pp. 2314-2318, 1979.

[24] G. Marquina, H. Waki, L. E. Fernandez et al., "Gangliosides expressed in human breast cancer," Cancer Research, vol. 56, no. 22, pp. 5165-5171, 1996.

[25] J. P. Oliva, Z. Valdés, A. Casacó et al., "Clinical evidences of GM3 (NeuGc) ganglioside expression in human breast cancer using the 14F7 monoclonal antibody labelled with 99mTc," Breast Cancer Research and Treatment, vol. 96, no. 2, pp. 115-121, 2006.

[26] A. M. Scursoni, L. Galluzzo, S. Camarero et al., "Detection of N-glycolyl GM3 ganglioside in neuroectodermal tumors by immunohistochemistry: an attractive vaccine target for aggressive pediatric cancer," Clinical and Developmental Immunology, vol. 2011, Article ID 245181, 6 pages, 2011.

[27] D. Osuna and E. de Álava, "Molecular pathology of sarcomas," Reviews on Recent Clinical Trials, vol. 4, pp. 12-26, 2009.

[28] E. G. Kanduma, J. C. Mukuria, and O. W. Mwanda, "Serum total sialic acid and Hanganutziu-Deicher antibody in normals and in cancer patients," East African Medical Journal, vol. 84, no. 5, pp. 207-214, 2007.

[29] P. Babál, P. Janega, A. Cerná, I. Kholováb, and E. Brabencovác, "Neoplastic transformation of the thyroid gland is accompanied by changes in cellular sialylation," Acta Histochemica, vol. 108, no. 2, pp. 133-140, 2006.

[30] V. Amberger-Murphy, "Hypoxia helps glioma to fight therapy," Current Cancer Drug Targets, vol. 9, no. 3, pp. 381-390, 2009.

[31] M. Nordsmark, J. Alsner, J. Keller et al., "Hypoxia in human soft tissue sarcomas: adverse impact on survival and no association with p53 mutations," British Journal of Cancer, vol. 84, no. 8, pp. 1070-1075, 2001.

[32] N. Burrows, M. Babur, J. Resch, K. J. Williams, and G. Brabant, "Hypoxia-inducible factor in thyroid carcinoma," Journal of Thyroid Research, vol. 2011, Article ID 762905, 17 pages, 2011.

[33] J. Yin, A. Hashimoto, M. Izawa et al., "Hypoxic culture induces expression of sialin, a sialic acid transporter, and cancerassociated gangliosides containing non-human sialic acid on 
human cancer cells," Cancer Research, vol. 66, no. 6, pp. 2937-2945, 2006.

[34] J. de Leòn, A. Fernández, C. Mesa, M. Clavel, and L. E. Fernández, "Role of tumour-associated N-glycolylated variant of GM3 ganglioside in cancer progression: e ffect over CD4 expression on T cells," Cancer Immunology, Immunotherapy, vol. 55, no. 4, pp. 443-450, 2006.

[35] J. de León, A. Fernández, M. Clavell et al., "Differential influence of the tumour-specific non-human sialic acid containing GM3 ganglioside on CD4+CD25- effector and naturally occurring $\mathrm{CD} 4+\mathrm{CD} 25+$ regulatory $\mathrm{T}$ cells function," International Immunology, vol. 20, no. 4, pp. 591-600, 2008. 


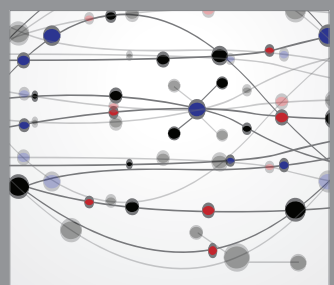

The Scientific World Journal
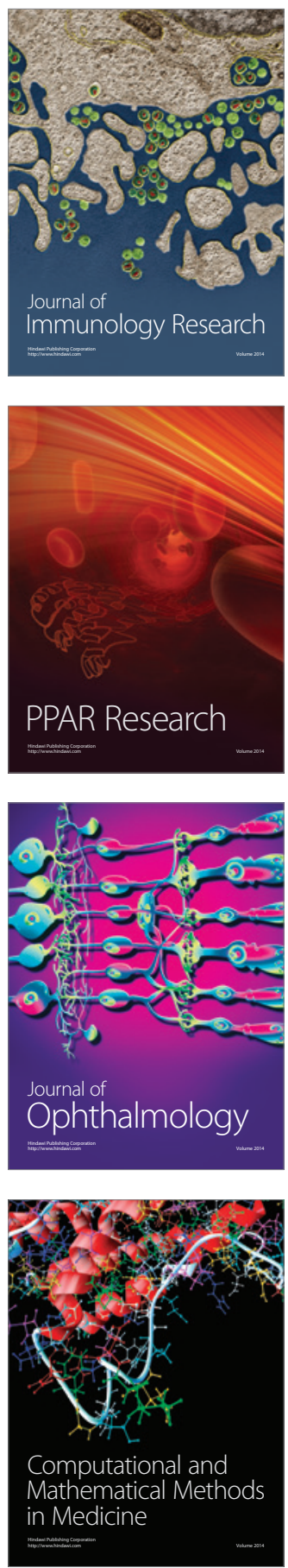

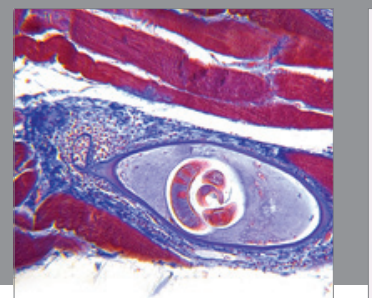

Gastroenterology

Research and Practice
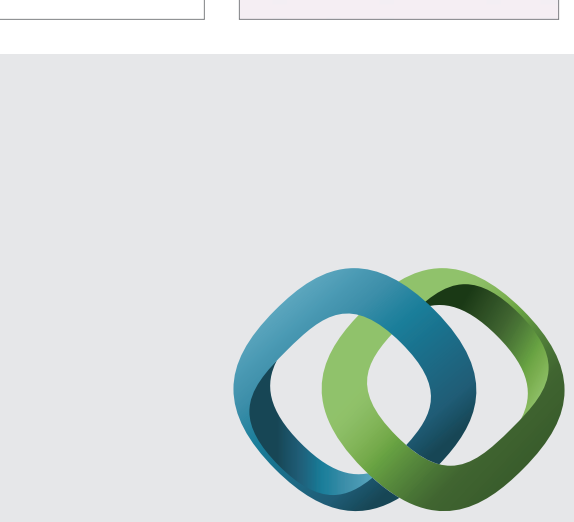

\section{Hindawi}

Submit your manuscripts at

http://www.hindawi.com
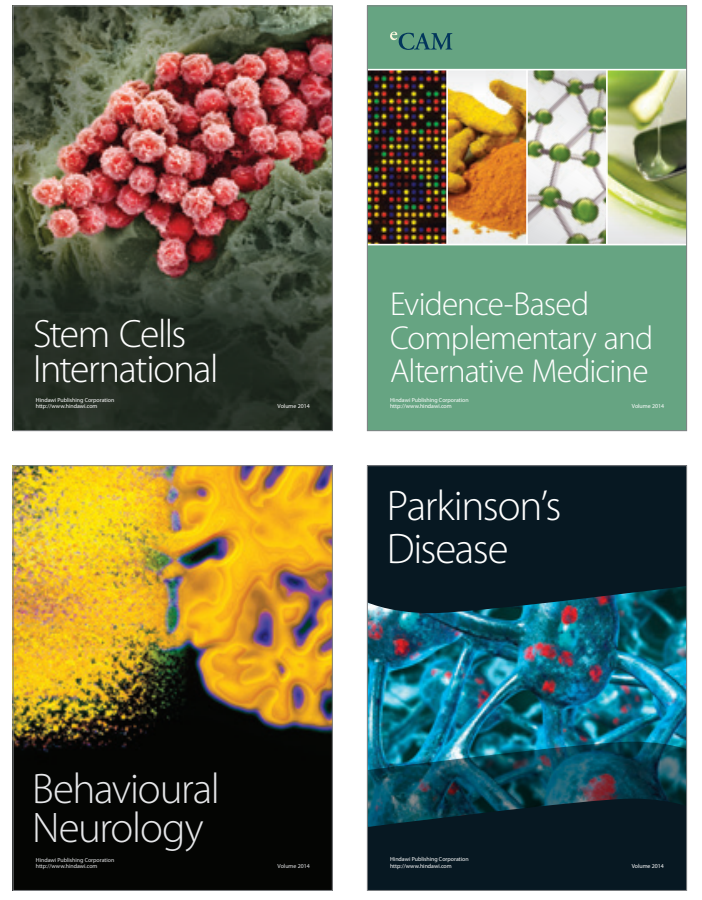
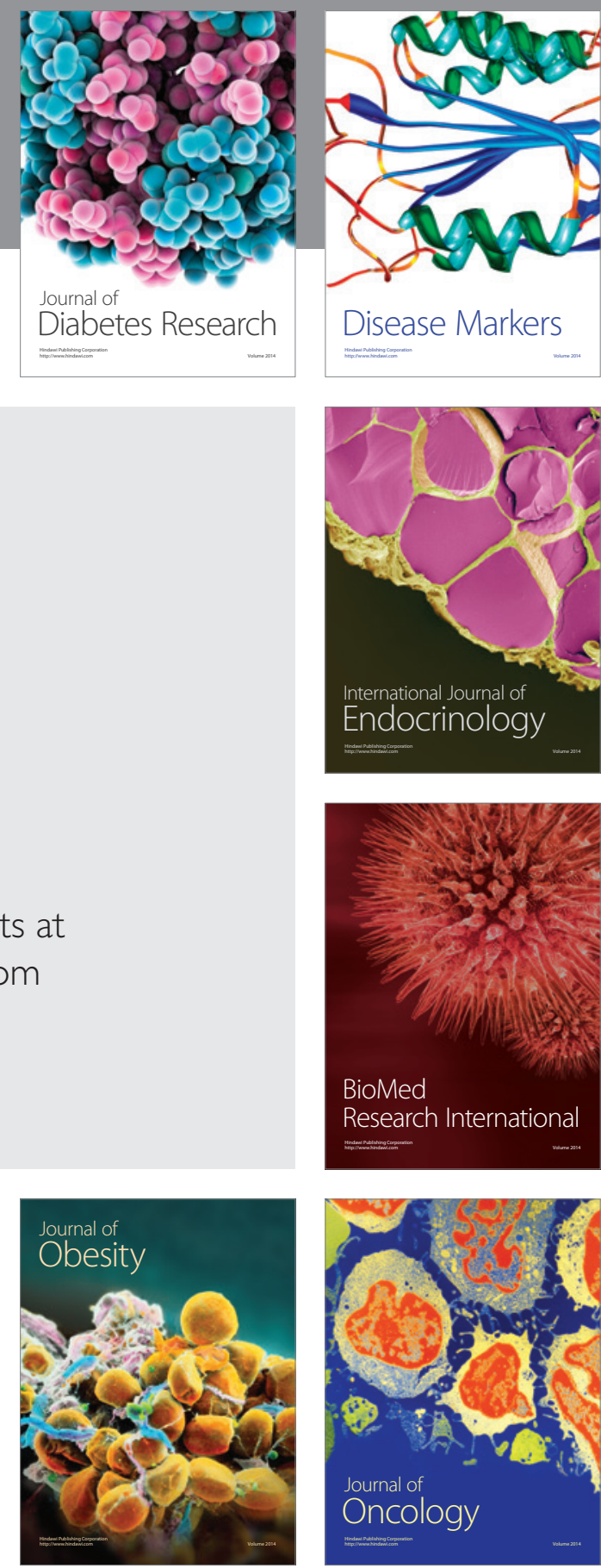

Disease Markers
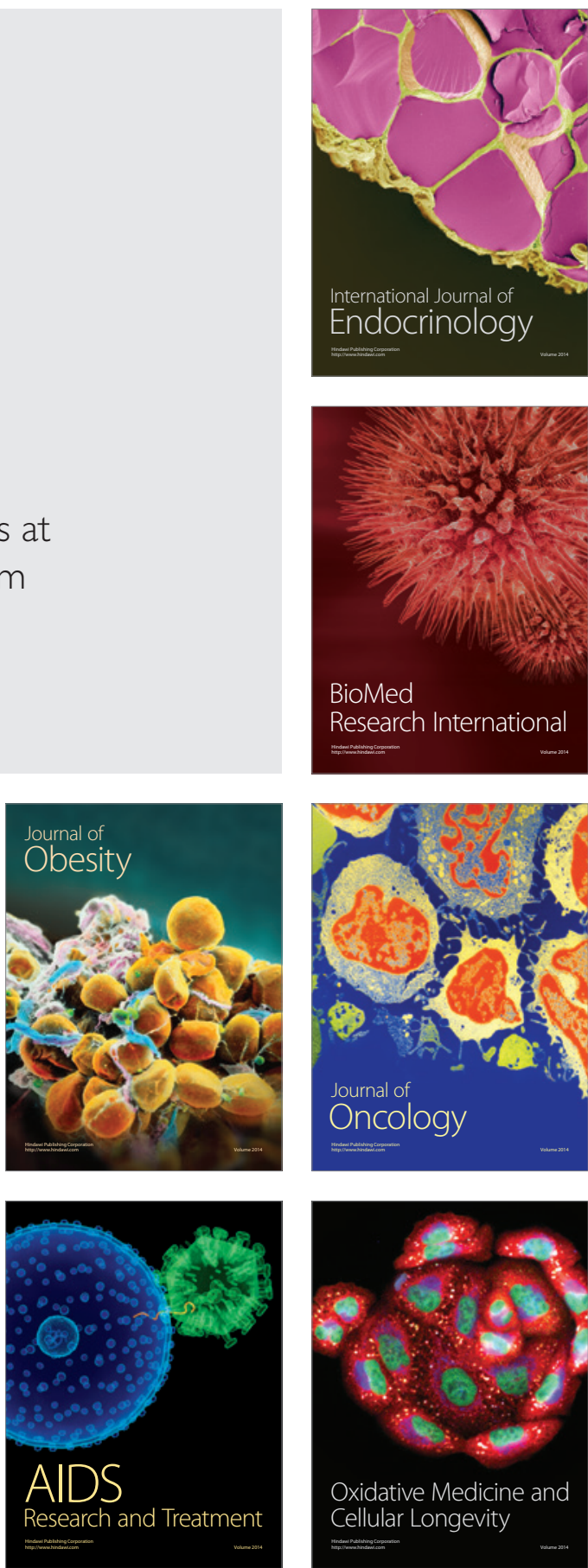